\title{
Retraction: Deconstructing the Ethernet
}

\author{
Zhong Chen and YongNing Guo \\ Department of Mathematics and Computer Science \\ Fuqing Branch of Fujian Normal University, Fuqing 350300, China \\ \{czcat, guoyn\}@163. com
}

Several conference proceedings have been infiltrated by fake submissions generated by the SCIgen computer program. Due to the fictional content the chapter "Deconstructing the Ethernet" by "Zhong Chen and YongNing Guo" has been retracted by the publisher. Measures are being taken to avoid similar breaches in the future.

D. Jin and S. Lin (Eds.): Advances in EECM Vol.2, LNEE 140, pp. 659-667. springerlink.com

(C) Springer-Verlag Berlin Heidelberg 2012 